\title{
Estudio piloto sobre la Comunicación y Transmisión del conocimiento Científico en los grupos de investigación universitarios
}

\author{
Pilot study on the communication and transmission of scientific \\ knowledge in the university research groups
}

Dra. Carmen López

Universidad de Alicante (UA). España

mc.lopez@ua.es

Dra. Mónica Gazquez Pertusa

Universidad Miguel Hernández de Elche (UMH). España

mgazquez@umh.es

Magister. Álvaro García del Castillo López

Universidad Miguel Hernández de Elche (UMH). España

alvaro.garciac@umh.es

Recibido: 13 de junio de 2011

Aceptado: 10 de septiembre de 2011

\begin{abstract}
Resumen
Los grupos de investigación universitarios comienzan a sentir la necesidad de gestionar la comunicación en el planteamiento, desarrollo y difusión de sus investigaciones. Es necesario poner en valor el uso de la comunicación en los programas científicos de actuación y sus resultados, como herramienta que contribuye a la eficacia, difusión y aplicación de los resultados de la investigación. En este estudio se presentan los resultados de la aplicación de un cuestionario a miembros de grupos de investigación universitarios, con el objetivo de conocer cómo gestionan la comunicación en sus proyectos, qué valor le atribuyen, qué tipo de actuaciones relacionadas con la comunicación realizan en cada etapa del proyecto y qué elementos condicionan la aplicabilidad de los resultados de investigación. Para ello, se indaga el uso habitual que se realiza de la comunicación en tres etapas: la comunicación para la investigación, la comunicación en la investigación y la comunicación de la investigación. Los resultados ponen de manifiesto la infrautilización de la comunicación en los procesos de investigación aplicados y en el establecimiento de interacciones entre el grupo investigador y los públicos involucrados o afectados por los resultados de la investigación. En conclusión, la gestión de la comunicación como herramienta de los grupos
\end{abstract}


de investigación asegura la eficacia académica y la calidad de las investigaciones, así como la aplicabilidad socioeconómica de los resultados de la investigación.

\section{Summary}

University research groups are beginnig to feel neccesary manage communication in planning, development and dissemination of his investigation. It's neccesary to value the use of communication in scientific programs and his results, as a tool wich contributes to efficacy, dissemination and aplication of investigation's results. In this study we present the results of a questionnaire application in a university research group members, with the aim to know how they manage communication in his projects, what value is attributed, what kind of actuations related to communication are done in the differents parts of the project and what elements are conditioning the applicability of research results. For that, we inquire what is the usual use of communication in three steps: communication for research, communication in research and communication of the research. Results show the underutilization of communication in processes of applied research and in the stablishment of interactions between the research group and public involved or afected by research results. In conclusion, communication management as a tool of the research groups, ensure the efficacy and quality of researchs and the socioeconomic applicability of research results.

Palabras Clave: Comunicación; Investigación; Ciencia

Key Words: Communication, Research, Science

\section{Introducción}

Desde el grupo de investigación de la universidad de Alicante "Comunicación y grupos específicos" se considera necesario abrir una línea de investigación orientada a estudiar el uso que se hace de la comunicación en el ámbito investigador, abarcando desde su inicio (antes de plantearse la investigación), a lo largo de la investigación en sí misma, y a la hora de transmitir los resultados, lo que se denomina transmisión del conocimiento generado. Creemos, al igual que otros autores, que dependiendo de los planteamientos de comunicación, tanto interna como externa, se mejorará la eficacia del proceso y de los resultados de las investigaciones. Pensamos que dicha eficacia afectará positivamente a la transmisión del conocimiento científico, que debería ser el fin último tanto de los grupos de investigación como de las instituciones financiadoras. Afortunadamente, en la actualidad se asume de forma indiscutible la relevancia que tiene el hacer partícipe a la sociedad de los avances de la ciencia y tecnología (Blanco, 2004). En esta línea son numerosas las razones que justifican esta necesidad como nos indica González-Alcaide (2009), basándose en Burkett (1973) y Junyent (2003), quien destaca dos razones fundamentales que motivan la 
necesidad de la divulgación científica: la enorme complejidad y especialización de la ciencia moderna, lo que hace necesaria la divulgación para que los propios científicos conozcan los avances en otros campos del saber, y, por otra parte, el gran influjo que la ciencia y la tecnología tienen en nuestras vidas cotidianas, convirtiendo la divulgación en una herramienta imprescindible para estar al día de los últimos avances. Por lo tanto, los conocimientos científicos deben ser accesibles y formar parte del acerbo cultural básico de cualquier persona. En la misma línea, Martín y Rey (2007: 17) nos indican que "los científicos constituyen un importante eslabón en la cadena de comunicación de la ciencia y transmisión del conocimiento científico. Sin embargo, no abundan los trabajos que aborden aspectos relacionados con el papel de la comunidad científica en la divulgación de la ciencia, sus patrones de comunicación con el público, o sus pautas de actuación y motivaciones, a la hora de participar en actividades divulgativas". Según Tena (2003: 190) "la información científica necesita de una permanente reclamación porque no encuentra los canales adecuados y precisos para llegar a la sociedad".

En este trabajo realizamos un estudio piloto a partir de los grupos de investigación universitarios de diferentes áreas de conocimiento y de varias universidades, tanto públicas como privadas, orientado a estudiar el uso que se hace de la comunicación en el ámbito investigador. El primer paso será conocer la práctica real y la consideración estratégica del diseño y planificación de las acciones comunicativas de cada uno de los grupos investigadores. Un desarrollo posterior podrá generar propuestas válidas que mejoren la transmisión del conocimiento científico y poder generalizar el uso eficaz de la comunicación, como herramienta dinamizadora de la investigación. Nuestros planteamientos futuros están orientados a trasladar la importancia que tiene tanto la planificación estratégica de la comunicación en los grupos como la visibilidad de los resultados científicos. Para ello es necesario detectar previamente si dentro de los grupos se planifican adecuadamente o no, conocer su realidad, si tienen en cuenta a los públicos a los cuales van a dirigir los resultados y si se tiene en cuenta sus intereses y necesidades a lo largo del proceso de la investigación; si se adaptan los contenidos, formas y lenguaje a los receptores últimos de las informaciones, con el fin de que la transmisión del conocimiento científico tenga aplicabilidad socioeconómica y susciten interés social.

La comunicación es de indiscutible ayuda en el ámbito investigador, a pesar de las dificultades de índole comunicativa que encuentra el investigador para alcanzar el reconocimiento de su labor científica, y conseguir la transmisión y proyección del conocimiento generado en sus investigaciones (GESCIT, 2007). Si, adicionalmente, la comunicación se gestiona de forma estratégica, sus efectos sobre la eficacia de la investigación se multiplican. La estrategia implica anticipación y una toma de decisiones de 
forma argumentada para lograr los objetivos, obtener mejores resultados y aumentar la probabilidad de aplicabilidad (Pérez, 2001). El término estrategia históricamente lo vemos relacionado con la estrategia militar, ya en los siglos VI y V antes de Cristo, de la mano del general chino Sun Tsu, en su famoso libro "El arte de la Guerra". En el ámbito de la política se expande de la mano de Maquiavelo en su obra "El príncipe". Adam Smith consigue su aplicación al saber económico, al igual que Peter Drucker al management o Ries y Trout al ámbito del marketing.

La estrategia en comunicación nos permitirá utilizar todos los recursos disponibles para asegurar que conseguimos hacer llegar los avances investigadores con eficacia a todos los públicos de interés, aportando un valor añadido. Es innegable, que para llegar a proponer cambios necesitamos saber más del entorno organizativo de los grupos de investigación y de la percepción, en relación a la comunicación de los propios investigadores, porque, hasta ahora, desconocemos si su actividad se dirige unidireccionalmente hacia los requerimientos impuestos por los objetivos prioritarios que estipulan las entidades financiadoras.

A la vista de lo expuesto, podríamos afirmar que existen motivos suficientes que justifican el estudio de la gestión de la comunicación en el seno de los grupos de investigación. Por tanto, el objetivo de este trabajo es analizar y describir el grado de conocimiento que tiene cada investigador acerca de la gestión de la comunicación en el ámbito de los proyectos que desarrolla. Examinando, a su vez, el uso estratégico que se realiza de esta herramienta, a lo largo de las etapas por las que transcurre la investigación: la comunicación para la investigación -la gestión estratégica de la comunicación con los públicos involucrados en la temática objeto de estudio-, la comunicación en la investigación -en el propio desarrollo de los proyectos de investigación- y la comunicación de la investigación -difusión de los resultados y divulgación científica-.

\section{Metodología}

\subsection{Estrategias metodológicas}

Se utilizó un diseño de corte transversal y descriptivo. Siguiendo a Abascal y Grande (2007), se optó por realizar una exploración previa con objeto de conseguir información primaria sobre el uso que se realiza de la comunicación en el seno de los grupos de investigación académicos. Este planteamiento nos permitirá identificar posibles causas o formular nuevas hipótesis explicativas que nos ayuden a avanzar en nuestro objeto de estudio y adaptarlo a diversos campos de investigación.

\subsection{Participantes}

Facultad de Ciencias de la Información - Universidad de La Laguna

Avenida César Manrique, s/n; Campus de Guajara

38071 La Laguna, Tenerife (Islas Canarias - España) 
Para captar a los encuestados se empleó la técnica "bola de nieve". De esta manera, cumplimentaron el cuestionario piloto un total de 51 investigadores integrados en grupos de investigación, pertenecientes a universidades nacionales e internacionales (89,4\% y 10,6\% respectivamente). Del total de la muestra un 95,7\% estaban adscritos a universidades públicas y un $4,3 \%$ a privadas.

La edad media de los encuestados fue 41,9 (dt.=8,8), correspondiéndose un 43\% con mujeres y un $57 \%$ con hombres. En referencia al nivel de formación académica un $86 \%$ poseen el grado de doctor y un 14\% son licenciados y/o graduados. Con respecto a la posición que los encuestados ocupan en el grupo de trabajo, la mayoría son miembros del equipo de investigación (56,9\%), un 31,4\% son directores o investigadores principales y el resto son investigadores contratados y becarios licenciados (un 5,9\% en ambos casos). En la Tabla I se presentan las características empleadas para la de identificación de los encuestados.

Tabla I. Características de identificación de la muestra.

\begin{tabular}{lc}
\hline & $\mathrm{N}=51$ \\
\hline Edad Media (DT) & $41,90(8,801)$ \\
Sexo Hombres (n) & $43,1(22)$ \\
\% Mujeres (n) & $56,9(29)$ \\
Formación Académica & \\
\% Licenciatura/Grado & $17 \%(7)$ \\
\% Doctorado & $86 \%(43)$ \\
Posición dentro del grupo de investigación & \\
\% Director o I.P. & $31,4(16)$ \\
\% Miembro del grupo & $56,9(29)$ \\
\% Investigador Contratado & $5,9(3)$ \\
\% Licenciado Becario & $5,9(3)$ \\
\hline
\end{tabular}

Fuente: elaboración propia 


\subsection{Instrumentos}

Para indagar sobre el uso habitual que se realiza de la comunicación en cada etapa del proyecto se construyó una encuesta, la "Escala sobre Comunicación y Transmisión del Conocimiento Científico". El instrumento de recogida de información se ajusta a lo que definen Grande y Abascal (2005:23) como "un conjunto articulado y coherente de preguntas para obtener la información necesaria a la hora de realizar una investigación". Permite trasladar el objetivo de la investigación a preguntas concretas. Además homogeníza la obtención de información, ya que todos los encuestados responden a las mismas preguntas y éstas son formuladas del mismo modo para todos. El instrumento también permite tratar los datos de forma rápida y con software informático.

En este estudio presentamos la prueba piloto de la escala sometida a screening por un grupo de investigadores, con el fin de eliminar ambigüedades y posibles preguntas superfluas, añadir preguntas relevantes, simplificar las preguntas difíciles, cambiar el orden de las preguntas para agilizar el flujo de respuestas y corregir la redacción de las preguntas que dificulten la comprensión.

En primer lugar se decidió qué tipo de información era necesaria, es decir, cómo se gestionaba la comunicación en las tres fase del proceso investigador. Las tres etapas condicionaron el tipo de preguntas que se confeccionaron y el orden de aparición de las mismas. Se recabó información sobre lo que los grupos ya hacían, sobre el conocimiento que existía acerca de la aplicación de la comunicación a este campo concreto y posibles actuaciones futuras que revelaran la tendencia hacia la gestión de la comunicación, donde poder realizar asesoramiento o recomendaciones de mejora, si hubiera lugar a ello. En segundo lugar, y tras la primera fase de valoración por parte de los expertos, se adecuó la encuesta y se diseñó para su realización on-line.

En cuanto al formato de la escala, finalmente se optó por un cuestionario autoadministrado y dirigido fundamentalmente a investigadores del ámbito universitario.

En las instrucciones se proporciona una definición breve y concisa del concepto comunicación y del concepto comunicación estratégica que pretenden situar al encuestado en los objetivos de la investigación. Estas definiciones eran necesarias por la elasticidad del término comunicación y las innumerables acepciones y aplicaciones que se pueden considerar.

El cuestionario recoge información de los aspectos siguientes: 
- Datos de identificación. Los primeras preguntas (0 a 5 ) pretenden ubicar las respuestas y poder cruzar los datos. Se obtuvieron datos: del centro de trabajo, la edad, el sexo, el nivel de formación académica y la posición dentro del grupo.

- Comunicación Percibida. Las preguntas 6 y 7 están enfocadas a la evaluación del nivel de comunicación percibida de forma individual y con el grupo de referencia.

- Uso estratégico de la comunicación. Se redactaron preguntas relacionadas con la comunicación en la primera etapa de la investigación ( 8,9 y 10) comunicación durante el desarrollo de la investigación (11 y 12) y la comunicación en la fase de difusión científica y divulgativa de los resultados de la investigación (13 y 14).

\subsection{Procedimiento}

El cuestionario se administró vía e-mail a una serie de sujetos para ver su aplicabilidad, adjuntando un texto explicativo en el cuerpo del mensaje del contenido del enlace donde se encuentra alojado el cuestionario. Dicho texto fue personalizado y revelaba la finalidad de la investigación, el carácter anónimo y las instrucciones necesarias para su cumplimentación. Como indican Schonlau, Fricker y Elliott (2002) una de las ventajas de la aplicación de cuestionarios por Internet es la rapidez. La aplicación de cuestionarios por esta vía es más rápida que por medios tradicionales, a la hora de preparar el número de documentos que se quieren repartir, en su distribución y en la cumplimentación. A su vez, se consigue abaratar costes en desplazamientos, correo o telefonía.

\subsection{Análisis de los resultados}

Los datos obtenidos de a través del cuestionario on-line se almacenaron en una matriz de datos empleando el programa IBM SPSS en su versión 19. Se llevaron a cabo análisis de frecuencias y se utilizaron estadísticos descriptivos para caracterizar a la muestra y al uso que los encuestados efectúan de la comunicación eficaz durante las tres fases: la comunicación para la investigación, la comunicación en la investigación y la comunicación de la investigación.

\section{Resultados}

En este apartado se describen el nivel de comunicación percibida de forma individual y con el grupo de referencia y el uso estratégico que los sujetos realiza en las tres etapas de 
investigación. El objetivo es perfilar cómo los grupos gestionan la comunicación en el contexto de una investigación.

\subsection{Evaluación del nivel de comunicación percibida}

En primer lugar, cuando se analiza el uso de la comunicación que de forma individual realizan los encuestados se destaca principalmente que un $74 \%$ ha seleccionado el empleo estratégico de la comunicación para transmitir adecuadamente la información dentro del grupo de investigación, un $70 \%$ también la utiliza para difundir los resultados una vez finalizada la investigación y un $66 \%$ la aplica durante el desarrollo del trabajo para involucrar a los destinatarios. La opción de planificar la comunicación antes de plantear la investigación ha sido seleccionada por un $42 \%$ de los encuestados. Finalmente, en $2 \%$ del total de la muestra declara no utilizarla para mejorar la eficacia de la investigación y otro $2 \%$ no la utiliza pero tener la intención de hacerlo en un futuro (ver tabla II).

Tabla II. Uso de la comunicación estratégica para obtener un mejor rendimiento

\begin{tabular}{lc}
\hline & $\%(\mathrm{n})$ \\
\hline Para transmitir adecuadamente la información dentro del grupo & $74(37)$ \\
La planifico antes de hacer el planteamiento de la investigación & $42(21)$ \\
$\begin{array}{l}\text { Durante el desarrollo de la investigación para involucrar a los } \\
\text { destinatarios }\end{array}$ & $70(33)$ \\
Al finalizar para dar a conocer los resultados & $2(1)$ \\
No la utilizo para mejorar la eficacia de mi investigación & $2(1)$ \\
No la utilizo, pero lo haré en un futuro & $2(1)$ \\
No sabe/No contesta & \\
\hline
\end{tabular}

Fuente: elaboración propia

En referencia al nivel de comunicación percibido entre los miembros del grupo de investigación, como puede observarse en la figura 1 en torno a un $37 \%$ lo califica como aceptable, un $27,5 \%$ como bueno, un $23,5 \%$ como muy bueno y un $11,8 \%$ opina que podría mejorarse. Situándose la mediana en la categoría bueno (Amplitud semi-intercuartil igual a $0,5)$. 
Figura l. Nivel de comunicación existente entre los miembros del grupo de trabajo.

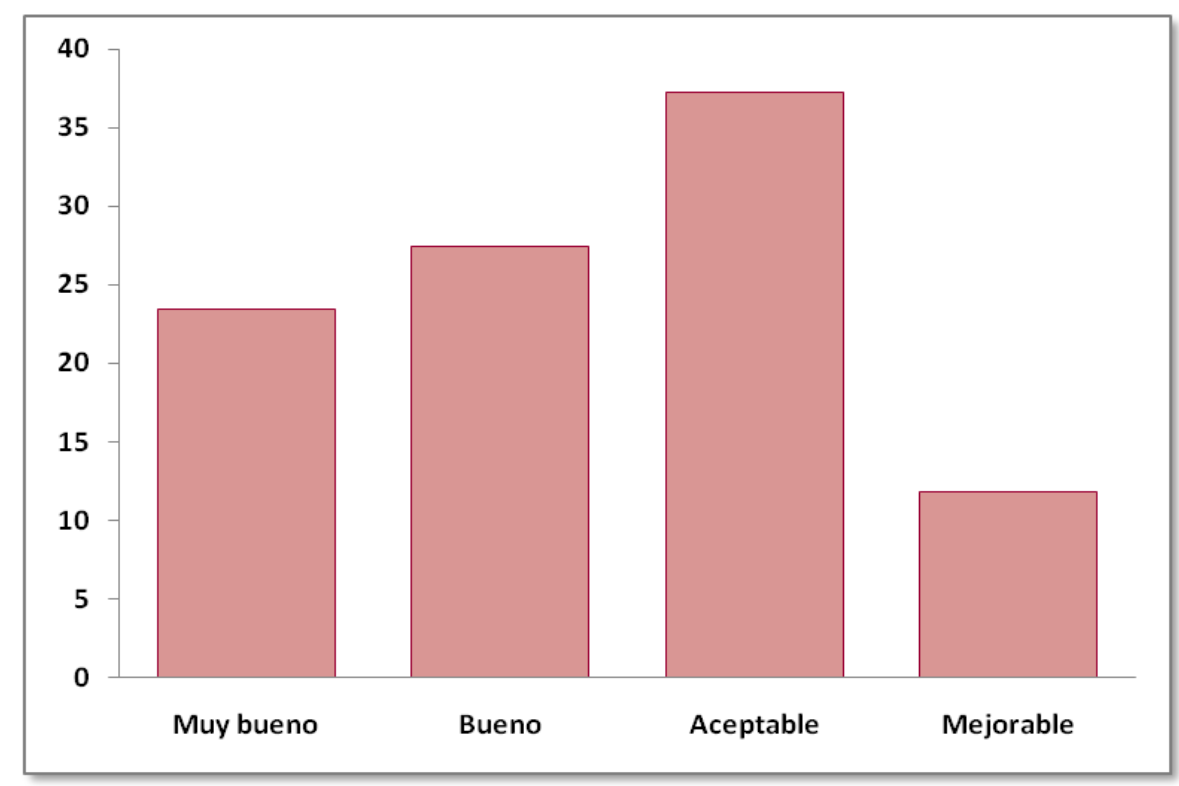

Fuente: elaboración propia

\subsection{Uso estratégico de la comunicación en la primera etapa de la investigación}

En cuanto al uso de la comunicación eficaz que los investigadores realizan en la primera etapa de la investigación (tal y como aparece en la tabla III) un 55,3\% de los encuestados afirma planificar la investigación considerando a los destinatarios pero sin realizar un contacto previo, un 38,3\% contacta con los destinatarios cuando planifica la investigación y tan sólo un 6,4\% afirma no considerar a los destinatarios.

Tabla III. Planificación de la investigación considerando a los destinatarios

\begin{tabular}{lr}
\hline & $\%(\mathrm{n})$ \\
\hline Sí, contacto con ellos previamente & $38,3(18)$ \\
Sí, pero no contacto con los destinatarios & $55,3(26)$ \\
previamente & \\
No & $6,4(3)$ \\
\hline
\end{tabular}

Fuente: elaboración propia 
Por otro lado, un $98 \%$ de los encuestados considera útil consultar las necesidades existentes en el ámbito de estudio antes de elegir el tema a investigar y un $2 \%$ no le concede utilidad. Sin embargo, un $19,6 \%$ declara no realizar habitualmente un estudio de necesidades al estar condicionado por las líneas prioritarias de la convocatoria en la que solicita información (ver tabla IV).

Tabla IV. Utilidad concedida a consultar las necesidades existentes en el ámbito de estudio

\begin{tabular}{lc}
\hline & $\%(\mathrm{n})$ \\
\hline Sí & $98(50)$ \\
No & $2(1)$ \\
Habitualmente no hago un estudio de necesidades & $19,6(10)$ \\
\hline
\end{tabular}

Fuente: elaboración propia

Por último, como puede apreciarse en la figura 2 al sondear con qué destinatarios se considera útil contactar antes de plantear la investigación se observa que la mayoría de los encuestados han seleccionado a los agentes públicos (68\%), a otros grupos de investigación relacionados con el tema (64\%) y a empresas privadas (58\%). Un $24 \%$ considera útil contactar previamente con población general, un $14 \%$ con consultoras públicas y privadas y un $2 \%$ con otros destinatarios.

Figura 2. Porcentaje de elección para la utilidad de contactar con diversos destinatarios

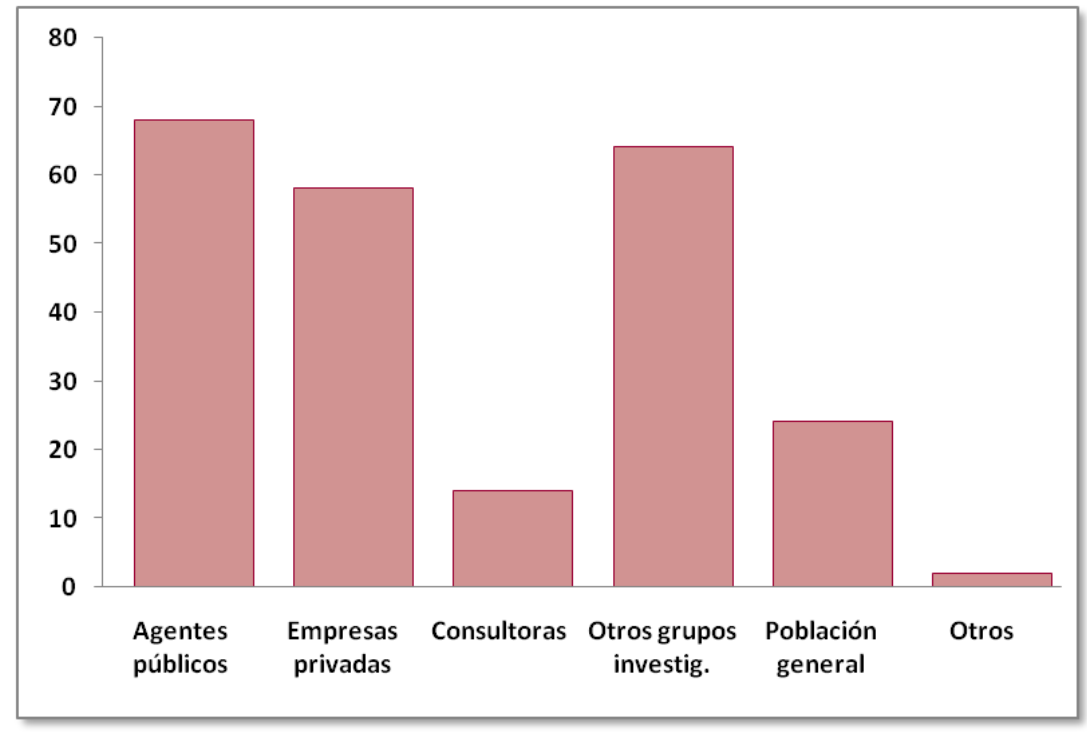

Fuente: elaboración propia 


\subsection{Uso estratégico de la comunicación durante el desarrollo de la investigación planteada por el grupo}

Como puede apreciarse en la tabla $\mathrm{V}$, un $74,5 \%$ de los encuestados considera útil comunicarse con los destinatarios involucrados durante la investigación frente a un $2 \%$ que no. Cabe destacar que en esta pregunta un $23,5 \%$ ha seleccionado la opción no sabe/no contesta.

Tabla V. Utilidad concedida a comunicarse con los destinatarios durante la investigación

\begin{tabular}{lc} 
& $\%(\mathrm{n})$ \\
\hline Sí & $74,5(38)$ \\
No & $2(1)$ \\
No sabe/No contesta & $23,5(12)$ \\
\hline
\end{tabular}

En la tabla VI aparecen las ventajas percibidas al contactar con los destinatarios durante el desarrollo de la investigación. A este respecto, destacan como principales ventajas conseguir involucrar a los destinatarios en la investigación $(68,6 \%)$, recabar su opinión sobre los objetivos de la investigación (56,9\%) y aprovechar los recursos con los que cuentan los destinatarios $(52,9 \%)$. La opción de obtener facilidades para el desarrollo de la investigación ha sido seleccionada por un $35,3 \%$ y tan sólo un $2 \%$ no encuentra ninguna ventaja en contactar con los destinatarios durante el desarrollo del trabajo investigador.

Tabla VI. Porcentaje de elección para las ventajas percibidas al contactar durante el desarrollo de la investigación con los destinatarios

\begin{tabular}{lc}
\hline & $\%(\mathrm{n})$ \\
\hline Conseguir involucrar a los mismos & $68,6(31,4)$ \\
Recabar su opinión sobre los objetivos de la investigación & $56,9(29)$ \\
Aprovechar los recursos con los que cuentan los destinatarios & $52,9(27)$ \\
Facilidades para el desarrollo de la investigación & $35,3(18)$ \\
No encuentro ventajas & $2(1)$ \\
No sabe/No contesta & $7,8(4)$ \\
\hline
\end{tabular}

Fuente: elaboración propia 


\subsection{Uso estratégico de la comunicación en la fase de difusión científica y divulgativa de los resultados de la investigación.}

Cuando se sondean los canales que con mayor frecuencia se utilizan para difundir los resultados de la investigación destacan principalmente los congresos o encuentros similares y los artículos en revistas científicas (84,3\% y $82,4 \%$ respectivamente). A mayor distancia se sitúa el empleo de las webs de los grupos de investigación $(37,3 \%)$ y los informes propios estipulados en las convocatorias $(21,6 \%)$. Los artículos en revistas divulgativas han sido seleccionados por un $15,7 \%$ y un $11,8 \%$ declara transmitir de forma personalizada los resultados a los interesados. Finalmente, las opciones de difusión de los resultados mediante blogs especializados y listas de distribución han sido seleccionadas por un 3,9\% de los encuestados (ver tabla VII).

Tabla VII. Porcentaje de elección para los canales más frecuentemente empleados para difundir los resultados

\begin{tabular}{lc}
\hline & $\%(\mathrm{n})$ \\
\hline Contacto con periodistas & $21,6(11)$ \\
Congresos o encuentros similares & $84,3(43)$ \\
Artículos en revistas científicas & $82,4(42)$ \\
A través de la web del grupo de investigación & $37,3(19)$ \\
En blogs especializados & $3,9(2)$ \\
Artículos en revistas divulgativas (orientadas al público en general) & $15,7(8)$ \\
A través de listas de distribución & $3,9(2)$ \\
Transmitiendo nuestros resultados en persona a los interesados & $11,8(6)$ \\
Los informes que estipule la convocatoria & $21,6(11)$ \\
\hline
\end{tabular}

Fuente: elaboración propia

Finalmente, el último aspecto valorado fueron las distintas maneras que los sujetos emplean para estructurar la información que desean trasmitir. Como se aprecia en la tabla VIII, los porcentajes son muy similares para las opciones cuidar el lenguaje para que sea entendible $(80,4 \%)$, redactarla en formato artículo $(78,4 \%)$ y adecuarla a los destinatarios a los que se dirigen $(74,5 \%)$. Seguidamente se sitúa la opción cuidar la forma para que sea atractiva y 
sencilla con un porcentaje de elección en torno al $61 \%$ y redactarla en formato informe de investigación 32,4\%. Tan sólo un 3,9\% de los encuestados declaran no tomar medidas especiales para estructurar la información.

Tabla VIII. Porcentaje de elección para las distintas maneras de estructurar la información a transmitir

\begin{tabular}{lc} 
& $\%(\mathrm{n})$ \\
\hline Cuido la forma para que sea atractiva y sencilla & $60,8(31)$ \\
Cuido el lenguaje para que sea entendible & $80,4(41)$ \\
Adecúo la información a los destinatarios a los que me quiero dirigir & $74,5(38)$ \\
La redacto en formato artículo de investigación & $78,4(40)$ \\
La redacto en formato informe de investigación & $31,4(16)$ \\
No tomo medidas especiales & $3,9(2)$ \\
\hline
\end{tabular}

Fuente: elaboración propia

\section{Discusión}

Los avances tecnológicos han permitido un incremento gradual del uso de medios informáticos en las diversas fases de contacto con el entrevistado, de recogida de la información, y de creación de la base de datos para el análisis de las respuestas. En nuestro caso la utilización del cuestionario on-line nos permite acceder a gran cantidad de investigadores abaratando los costes de la aplicación, simplificando el proceso y agilizando la participación.

Estamos convencidos de que este tipo de enfoques redunda en el avance del conocimiento científico, dado que uno de los pasos más cuestionados, como hemos mencionado anteriormente, es que los frutos de la investigación sean realmente aplicables y que sirvan para que la sociedad entienda y comparta sus metas.

Este estudio exploratorio constituye una aproximación inicial sobre el análisis del empleo y gestión que se realiza de la comunicación en el marco de la actividad investigadora. Los resultados obtenidos corroboran un adecuado manejo de la comunicación entre los integrantes del grupo de investigación. Así mismo, se verifica también su eficacia al finalizar la investigación para difundir los resultados, con objeto de obtener un mejor rendimiento. En general, en la fase de planificación de la investigación la mayoría de los encuestados conceden importancia a la realización de una evaluación de necesidades en el ámbito de 
estudio, pero sólo la mitad contacta con los destinatarios previamente. Por otro lado, se concede mayor importancia a contactar con agentes públicos, otros grupos de investigación y empresas privadas frente a otros destinatarios. En lo que respecta a la fase de desarrollo la mayoría de los encuestados encuentra útil realizar un contacto con los destinatarios con la finalidad de conseguir involucrarlos, valorar los objetivos propuestos y utilizar los recursos con los que cuentan los destinatarios. En la última fase de la investigación los encuestados prefieren difundir sus resultados mediante canales propios del ámbito científico-académico (artículos, congresos), detectándose una importante laguna en la transferencia de resultados hacia los destinatarios involucrados y la población general.

En definitiva, el estudio de la comunicación en todas sus vertientes se ha hecho un hueco fundamental en todas las disciplinas del conocimiento, porque se ha comprobado ampliamente que si funciona mal o no funciona, los resultados se ven mermados, llegando incluso a desacreditar y/o invalidar la eficacia esperada. Por ello pensamos que incluir esta visión enriquece aún más las posibilidades potenciales de cualquier proyecto, además de proporcionar nuevas vías de entendimiento entre los que lo desarrollan, los que lo promocionan y los que disfrutan de sus beneficios finales.

Con toda seguridad, a partir de planteamientos de este corte, los propios implicados serán los que puedan comprobar que se mejora significativamente utilizando la comunicación estratégica y serán los que soliciten una mayor formación en este campo de acción.

Somos conscientes de que en este estudio piloto existen una serie de limitaciones que tendremos en cuenta a la hora de proseguir con este proceso: la multidisciplinariedad de los grupos, los enfoques de investigación y la interrelación con los públicos objetivo. Además a partir del cuestionario del que partimos, habrá que filtrar muchos de los ítems, mejorar los que aparezcan como poco relevantes, eliminar la sobre explicación cuando sea necesario y añadir aquellos aspectos que se hayan quedado fuera del espectro que queremos evaluar.

\section{Referencias}

Abascal, E. y Grande, I. (2007): Fundamentos y técnicas de investigación comercial. Madrid: Esic.

Blanco, A. (2004): "Relaciones entre la educación científica y la divulgación de la ciencia". Revista Eureka sobre Enseñanza y Divulgación de las Ciencias, 1(2), pp. 70-86.

Burkett, D. W. (1973): Writing science news for the mass media. Houston (Texas): Gulf Publishing Company. 
GESCIT (Grup d'Estudis Socials de la Ciència i la Tecnologia) (2007): "Análisis de los factores psicosociales que inciden en la difusión y proyección de la actividad científica española. Informe del estudio cualitativo previo". Athenea Digital, 12, pp. 183-239.

González-Alcaide, G. et al. (2009): "La investigación sobre la divulgación de la ciencia en España: Situación actual y retos para el futuro". ARBOR Ciencia, Pensamiento y Cultura, 738, julio-agosto, pp. 861-869.

Grande, I, y Abascal, E. (2005): Análisis de encuestas. Madrid: ESIC.

Junyent, C. (2003): "Comunicació científica i percepció social de la Ciencia". Coneiximent $i$ Societat: Revista d'Universitats, Recerca i Societat de la Informació, 2, pp. 6-15.

Martín, M.J. y Rey, J. (2007): El papel de los científicos en la comunicación de la ciencia y la tecnología a la sociedad: Actitudes, aptitudes e implicación. Comunidad de Madrid: Consejería de Educación, Dirección General de Universidades e Investigación.

Pérez, R.A. (2001): Estrategias de Comunicación. Barcelona: Ariel.

Schonlau, M., Fricker, R.D. y Elliott, M.N. (2002): Conducting research surveys via e-mail and the Web. Santa Monica, CA: Rand.

Sun Tsu (2006): El arte de la guerra. Versión de Thomas Cleary. Madrid: EDAF.

Tena, J. (2003): "La Ciencia desaparecida". Revista Iberoamericana de Ciencia, Tecnología y Sociedad (CTS), no 1, vol 1, septiembre, pp, 190.

\section{Forma de citar este artículo en bibliografías}

LOPEZ, C.; GAZQUEZ PERTUSA, M. y GARCIA DEL CASTILLO LOPEZ, A. (2011): "Estudio piloto sobre la Comunicación y Transmisión del conocimiento Científico en los grupos de investigación universitarios", en Revista PANGEA, 2, páginas 32 a 46 . Red Académica Iberoamericana de Comunicación. Recuperado el _ de __ de 2 ___ de: http://revistapangea.org 